\title{
Toward the Monitoring of Blazars at Abastumani
}

\author{
O. M. Kurtanidze \\ Abastumani Astrophysical Observatory, Abastumani, 83762, Georgia
}

\begin{abstract}
We give a brief summary of the ongoing Abastumani Blazar Monitoring Program (ABMP) started in May 1997. More than 50,000 frames of data have been collected during 370 nights of observations on about 50 target objects. Preliminary results of two years of monitoring of ten selected AGNs carried out in the framework of ABMP are presented. All observations were done in the BVRI bands using the CCD ST-6 based photometer attached to the 70-cm meniscus telescope's Newtonian focus. Image reductions were made using different software packages of image reduction systems such as IRAF, MIDAS and STARLINK. All objects under study show light variations exceeding one magnitude. The largest variation was observed for $\mathrm{AO} 0235+116$, amounting to $4.0 \mathrm{mag}$ in the $\mathrm{R}$ band.
\end{abstract}

\section{Discussion}

Donald Lynden-Bell: Do you have colour variations too?

Omar Kurtanidze: This is a very interesting question. Yes we have. From a preliminary analysis we were able to conclude that colour variations depend on the activity state, i.e. they are larger at higher activity.

Charley Lineweaver: Do you monitor non-variable stars to calibrate the variation - correct for seeing, etc.?

Kurtanidze: In most cases there are a lot of stars on a frame to check for possible false variability caused by second order extinction or short-term variability of comparison stars. The final light curve is a mean relative to three comparison stars.

Brian McBreen: What was the fastest light variation observed with your large monitoring program?

Kurtanidze: In the low state of BL Lacertae, the fastest variation is 0.30 mag in a hundred minutes, while variations of 0.10 to 0.15 mag in 60 minutes are very common. They could be twice as large in the high state. 